\title{
Treatment Principles of Obesity with Chinese Herbal Medicine: Literature Analysis by Text Mining
}

\author{
Yunyu Huang ${ }^{1 *}$, Lianjie Wang ${ }^{{ }^{*}}$, Shidong Wang ${ }^{1}$, Feng Cai ${ }^{2}$, Guang Zheng ${ }^{3}$, Aiping Lu $^{2,4}$, \\ Xiuchen $\mathrm{Yu}^{1 \#}$, Miao Jiang ${ }^{2 \#}$ \\ ${ }^{1}$ Beijing Dongzhimen Hospital affiliated to Beijing University of Chinese Medicine, Beijing, China \\ ${ }^{2}$ Institute of Basic Research in Clinical Medicine, China Academy of Chinese Medical Sciences, Beijing, China \\ ${ }^{3}$ School of Information Science-Engineering, Lanzhou University, Lanzhou, China \\ ${ }^{4}$ Hong Kong Baptist University School of Chinese Medicine, Kowloon, Hong Kong, China \\ Email: \#yuxiuch@sina.com, \#miao_jm@126.com
}

Received September 2012

\begin{abstract}
Obesity represents a social health problem worldwide, associated with serious health risks and increased mortality. The prevalence of obesity is reported to be increasing in both developed and developing countries. Obesity is associated with a significant range of comorbidities and is linked with increases in mortality, thus the treatment of obesity is very important. Chinese herbal medicine (CHM) has been used for weight management both in China and in western countries for many years, the effectiveness and safety of CHMs in obesity have been proved. Yet the principles of treating obesity with CHMs are hard to manage due to the complexity of TCM theory. In this study, a novel text mining method was developed based on a comprehensive collection of literatures in order to explore the treatment principles more intuitively. Networks of TCM patterns and CHMs which are most frequently used in obesity treatment are built-up and analyzed, two major principles are explored in treating obesity: one is resolving phlegm and dampness, the other is clearing heat and reinforcing deficiency. These findings might guide the clinicians in treatment of obesity.
\end{abstract}

Keywords: Obesity; Chinese Herbal Medicine; Pattern; Traditional Chinese Medicine; Text Mining

\section{Introduction}

Obesity [defined as a body mass index (BMI) $\geq 30 \mathrm{~kg} / \mathrm{m}^{2}$ ] represents a considerable worldwide health problem, associated with serious health risks and increased mortality. The prevalence of obesity is reported to be increasing worldwide [1]. At present, 35.5\% among adult men and $35.8 \%$ among adult women in the United States are considered to be obese [2], in England the prevalence of obesity is reported to have increased between 1993 and 2004 from $13.6 \%$ to $24.0 \%$ among men and from $16.9 \%$ to $24.4 \%$ among women. Some minority groups, such as Hispanic and African Americans, have higher overweight and obesity rates than the white population [3]. In line with the global trend, the rate of obesity in China continues to increase, overweight and obesity also pose a challenge to public health in China. According to Chinese definition, 303 million Chinese are over-weight (body mass index, BMI $\geq 24 \mathrm{~kg} / \mathrm{m}^{2}$ ). Among them, 73 million are clinically obese (BMI $\geq 28 \mathrm{~kg} / \mathrm{m}^{2}$ ) [4].

Obesity is associated with a significant range of comorbidities and is linked with increases in mortality, which

\footnotetext{
"Equal contributions to this work.

${ }^{\#}$ Corresponding author.
}

is usually the result of the combination of genetic factors and an inappropriate lifestyle, characterized by inadequate nutrition and lack of regular physical activity. It is closely associated with the development of type 2 diabetes, hypertension, dyslipidemia, and cardiovascular disease, among other medical problems [5].

Expert panels sponsored by both the World Health Organization and the National Institutes of Health have recommended that obese adults, as well as those who are overweight and have comorbid conditions, lose $10 \%$ of their initial weight $[6,7]$. A comprehensive program of lifestyle modification is considered the first option for achieving this goal [7]. Lifestyle modification, also referred to as behavioral weight control, includes 3 primary components: diet, exercise, and behavior therapy [8].

For obese patients who cannot achieve or maintain a healthy weight by non-pharmacological means, drug therapy is recommended in combination with non-pharmacological interventions such as dietary modifications and exercise [9]. Yet the costs are high. Compared with lifestyle advice, the mean incremental cost-effectiveness ratio for orlistat (sibutramine, rimonabant) ranged between $£ 970$ (£6941, £9303) and £59,174 (£10,042, 


\section{$£ 35,876)[9]$.}

Despite these conditions, patient surveys indicate that less than one-half of obese individuals are advised by their physicians to lose weight [10], the reason is possibly lying in that physicians feel ill-equipped to provide treatment or that the available treatments for obesity are ineffective [11].

Chinese herbal medicine (CHM) has been used for weight management both in China and in western countries, traditional Chinese medicine (TCM) has a distinctive and systematic cognition of obesity according to the specific theory framework. Randomized, controlled clinical trials published on CHMs for weight management have proved the effectiveness and safety of some CHM formulae [12,13]. A number of animal studies support the use of CHM formulas for treating obesity and have shown other beneficial effects [14-16], the potential mechanism of actions of many Chinese herbs that are traditionally used for weight management has also been explored.

However, due to the complexity of TCM theory, the treatment principles of obesity are complicated and mysterious. In order to explore the treatment principles more intuitively, a novel text mining method was developed based on a comprehensive collection of literatures [17]. The study would provide an accessible way for understanding the treatment principles for obesity with CHMs.

\section{Material and Methods}

\subsection{Data Collection}

The dataset were downloaded from SinoMed

(http://sinomed.cintcm.ac.cn/index.jsp) with the query term of "obesity" on Jane 22, 2012. This dataset contains 38,051 records of literatures on clinical practices or theoretical research on obesity. In this dataset, each record/paper is tagged with an unique ID. These records contain the title, keywords, and abstract of published papers [17].

\subsection{Data Filtering}

1) TCM Patter: Pattern (also called as Syndrome, or Zheng) differentiation is regarded as the key role in the clinical practise of TCM traditional Chinese medicine [18]. Usually, pattern identification is the basis of the prescription of herb formulae, CHMs, or other TCM therapies. Thus it is natural and intuitive to filter out the pattern and then try to find the association rules between pattern and CHMs. The top TCM patterns in obesity are: pulmonary stagnation of phlegm (Tan shi zu fei), followed with stagnation of liver qi (Gan qi yu jie) and kidney yin deficiency (Shen yin $x u$ ).

2) Chinese herbal medicine: Based on the keyword list of CHMs (both legal names and other popular names are included for calculation), we filtered the CHMs in the plain text format, and then converted all popular names into legal names. All the CHMs were tagged with their unique paper ID. Based on the unique paper ID, we could construct the pairs of co-existed CHMs as they do coexisted in literature. For example, in one paper, CHMs of Huangqi (Radix Astragali seu Hedysari), Renshen (Radix Ginseng), and Shengdihuang (Radix Rehmanniae Recens) are mentioned. Then, the pairs of co-existed CHMs of "Huangqi-Renshen", "Huangqi-Shengdihuang”, and "Renshen-Shengdihuang” are constructed.

\section{Results}

In this paper, focused on obesity, we explored the principles of pattern differentiation and CHMs prescription and the association between the two aspects under the framework of TCM theory from 38,051 literatures. The network construction is based on the analysis of networks of pattern and CHM correlated with obesity in literature. The connections among these networks are built-up under the professional knowledge of TCM.

\subsection{Major TCM Patterns in Obesity}

Pattern identification is regarded as the first step during TCM clinical practice procedure. After the pattern is approved, the treatment principle can be determined. For example, when the pattern of blood stasis is approved, then the treatment principle of active blood and resolve stasis is determined. In our results, 122 TCM patterns are detected to be related with obesity, and the top 10 TCM patterns in obesity are presented in Figure 1.

\subsection{Most Frequently Prescribed CHMs in Obesity Treatment}

Altogether 174 CHMs are mined from the literature in treatment of obesity. As herbal formulae are composed by the CHMs, the list of most frequently used CHMs can certainly provide the information of TCM treatment principles more effectively due to the stablity and uniqueness of each CHMs rather than formulae which can be renamed easily after slight regulation. The top 10 frequently prescribed CHMs are shown in Figure 2.

\subsection{Networks of the Pattern and CHMs in Obesity}

The networks of patterns and CHMs in obesity treatment can be constructed based on the co-existence frequency among patterns or CHMs, respectively. By checking these two networks, the correlation between TCM patterns and CHMs can be analyzed and explored. In order 


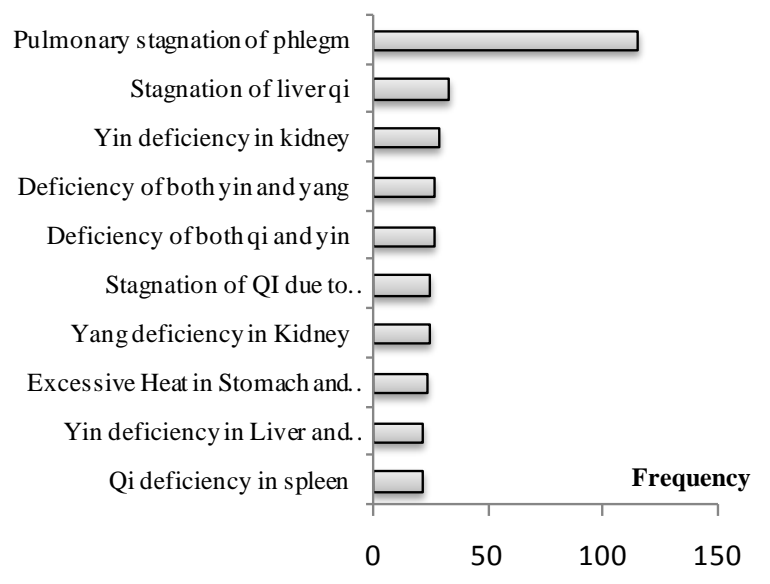

Figure 1. Top 10 TCM patterns in obesity.

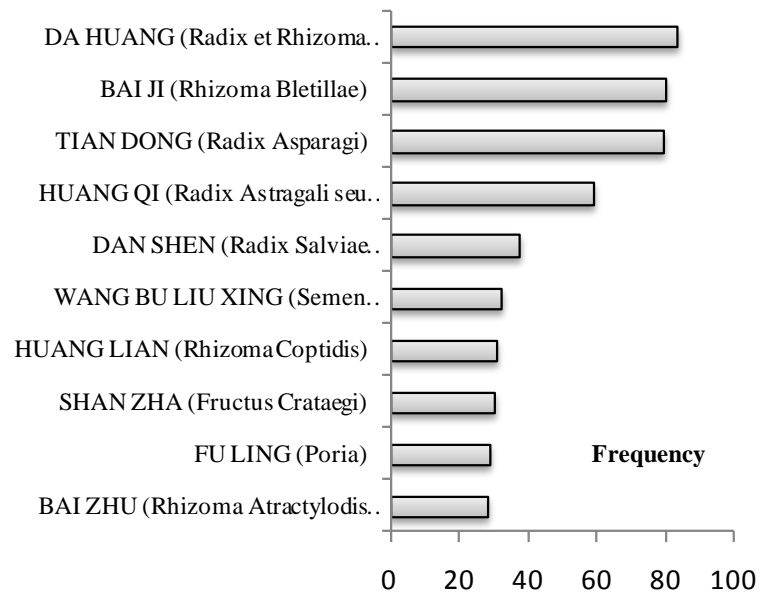

Figure 2. Top 10 frequently prescribed CHMs in treatment of obesity. to achieve better visualization, the CHM network is simplified to preserve $11 \mathrm{CHMs}$ which are the most frequently used in combination in treating obesity. The networks of pattern and CHMs with their correlation on obesity is demonstrated in Figure 3. The major correlation between TCM patterns identification and CHMs are demonstrated with arrows.

\section{Conclusions and Discussion}

Based on the analysis described in previous section, it naturally comes to the point that TCM treatment principles of a disease can be reasonably mined out and presented from dataset downloaded from SinoMed which contains 38,051 records. Compared with the knowledge of obesity in textbook, most knowledge is covered by the simple and succinct networks demonstrated in Figure 3 which can be summarized with the following points and their internal connections.

1) TCM networks of patterns and CHMs can be constructed and analyzed

In this study, through mass calculation on dataset on obesity, the main aspects of TCM networks were built-up. The main TCM organs (different from modern medical concept) involved in obesity development are lung, spleen, liver, kidney and stomach. The pathogenesis related with obesity includes Dampness-Phlegm, Qi-depression, Deficiency of Qi/Yin/Yang, and Pathogenic fire. To follow the matter of course, CHMs most frequently prescribed in obesity treatment can be grouped into 2 major classes, one group is responsible for resolving phlegm and dampness, the other is for clearing heat and reinforcing deficiency. These major principles might

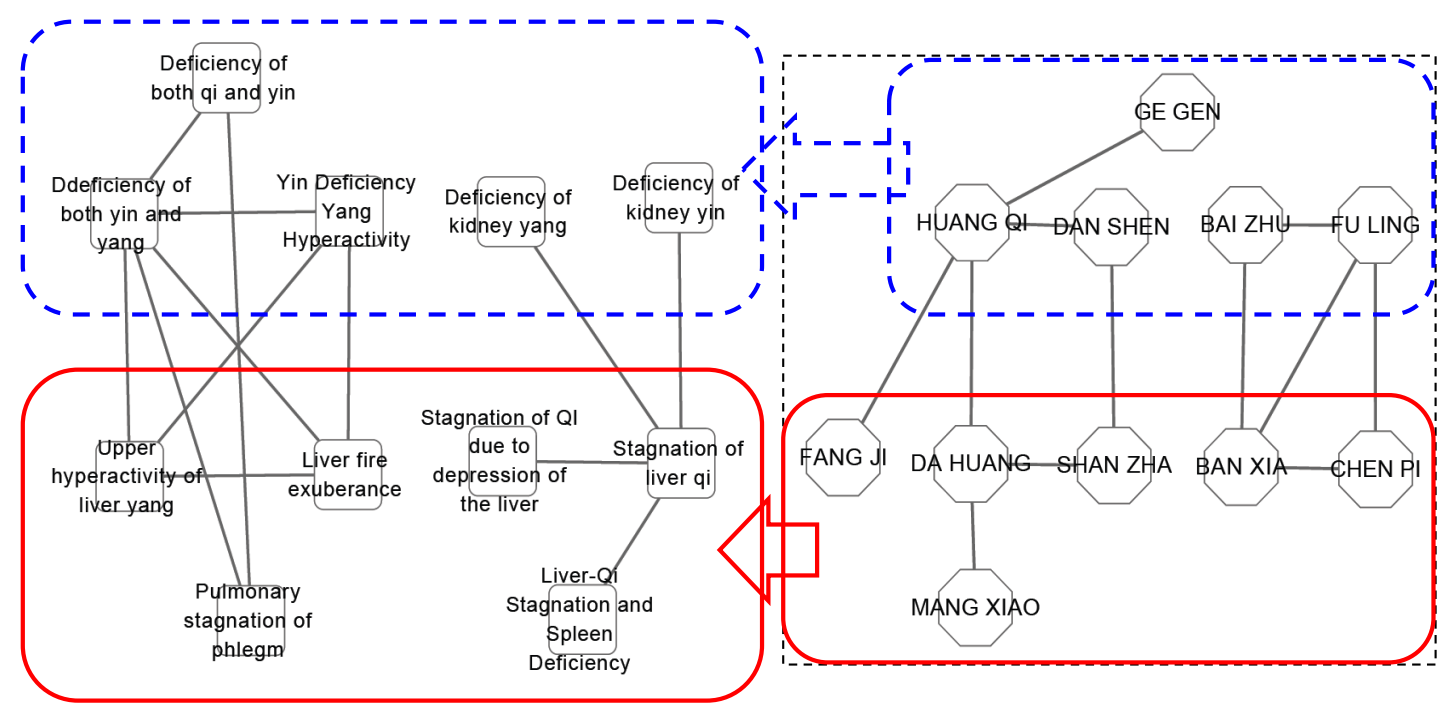

Figure 3. The network of TCM patterns and CHMs in treatment of obesity. Network of patters is shown in the upper part, network of CHMs in lower part. Bigger shape represents higher frequencies. The lines between the shapes represent the co-existent correlations between the two patterns/CHMs. Arrows represent the correlation between the TCM patterns and CHMs. 
guide the clinicians in treatment of obesity.

\section{2) Internal connections among networks}

Through directed text mining, the internal connections among TCM networks were also found. These internal connections can be grouped into two major hierarchical clusters. Each cluster is associated with one major kind of patterns. The major treatment principles of TCM treatment of obesity can be explored by text mining method and summarized in a succinct figure.

3) TCM Network might be useful in both TCM clinical practices and scientific researches

The network demonstrated in Figure $\mathbf{3}$ can be taken as a high level of abstraction on the treatment of obesity out of dataset containing 27,442 records. From the view point of clinicians, it can be taken as a kind of reference. From the view point of basic researchers, this result might be useful to illuminate some further studies in obesity.

\section{Acknowledgements}

This work was partially supported by National Science Foundation of China (No. 30902003 and 81072982). 2012' Traditional Chinese medicine Professional project (No. 201207012).

\section{REFERENCES}

[1] A. E. Caballero, "Endothelial Dysfunction in Obesity and Insulin Resistance: A Road to Diabetes and Heart Disease," Obesity Research, Vol. 11, No. 11, 2003, pp. 12781289. http://dx.doi.org/10.1038/oby.2003.174

[2] K. M. Flegal, M. D. Carroll, B. K. Kit and C. L. Ogden, "Prevalence of Obesity and Trends in the Distribution of Body Mass Index among US Adults, 1999-2010,” JAMA, Vol. 307, No. 5, 2012, pp. 491-497. http://dx.doi.org/10.1001/jama.2012.39

[3] K. M. Flegal, M. D. Carroll, C. L. Ogden and L. R. Curtin, "Prevalence and Trends in Obesity among US Adults, 1999-2008,” JAMA, Vol. 303, No. 3, 2010, pp. 235-241. http://dx.doi.org/10.1001/jama.2009.2014

[4] Y. Gao, S. Griffiths and E. Y. Chan, "Community-Based Interventions to Reduce Overweight and Obesity in China: A Systematic Review of the Chinese and English Literature," Journal of Public Health (Oxford), Vol. 30, No. 4, 2008, pp. 436-448. http://dx.doi.org/10.1093/pubmed/fdm057

[5] F. X. Pi-Sunyer, "The Obesity Epidemic: Pathophysiology and Consequences of Obesity," Obesity Research, Vol. 10, No. Suppl 2, 2002, pp. 97S-104S. http://dx.doi.org/10.1038/oby.2002.202

[6] "Obesity: Preventing and Managing the Global Epidemic. Report of a WHO Consultation,” World Health Organization Technical Report Series, Vol. 894, 2000, pp. i-xii, $1-253$.

[7] "Clinical Guidelines on the Identification, Evaluation, and Treatment of Overweight and Obesity in Adults-The Evidence Report. National Institutes of Health, Obesity
Research, Vol. 6, No. Suppl 2, 1998, pp. 51S-209S. http://dx.doi.org/10.1002/j.1550-8528.1998.tb00690.x

[8] "Clinical Guidelines on the Identification, Evaluation, and Treatment of Overweight and Obesity in Adults," WMJ, Vol. 79, No. 9, 1998, pp. 20-21, 24-25, 27-37.

[9] R. Ara, L. Blake, L. Gray, M. Hernandez, M. Crowther, A. Dunkley, F. Warren, R. Jackson, A. Rees, M. Stevenson, et al., "What Is the Clinical Effectiveness and Cost-Effectiveness of Using Drugs in Treating Obese Patients in Primary Care? A Systematic Review,” Health Technology Assessment Journal, Vol. 16, No. 5, 2012, pp. iii-xiv, 1195.

[10] D. A. Galuska, J. C. Will, M. K. Serdula and E. S. Ford, "Are Health Care Professionals Advising Obese Patients to Lose Weight?” JAMA, Vol. 282, No. 16, 1999, pp. 1576-1578. http://dx.doi.org/10.1001/jama.282.16.1576

[11] J. E. Harris, V. Hamaday and E. Mochan, "Osteopathic Family Physicians' Attitudes, Knowledge, and Self-Reported Practices Regarding Obesity," The Journal of the American Osteopathic Association, Vol. 99, No. 7, 1999, pp. 358-365.

[12] G. B. Lenon, K. X. Li, Y. H. Chang, A. W. Yang, C. Da Costa, C. G. Li, M. Cohen, N. Mann and C. C. Xue: "Efficacy and Safety of a Chinese Herbal Medicine Formula (RCM-104) in the Management of Simple Obesity: A Randomized, Placebo-Controlled Clinical Trial,” EvidenceBased Complementary and Alternative Medicine, Vol. 2012, 2012, Article ID: 435702.

[13] C. Hioki, K. Yoshimoto and T. Yoshida, "Efficacy of Bofu-Tsusho-San, an Oriental Herbal Medicine, in Obese Japanese Women with Impaired Glucose Tolerance," Clinical and Experimental Pharmacology and Physiology, Vol. 31, No. 9, 2004, pp. 614-619. http://dx.doi.org/10.1111/j.1440-1681.2004.04056.x

[14] N. A. Talpur, B. W. Echard, V. Manohar and H. G. Preuss, "Influence of a Combination of Herbs on Appetite Suppression and Weight Loss in Rats," Diabetes, Obesity and Metabolism, Vol. 3, No. 3, 2001, pp. 181-185. http://dx.doi.org/10.1046/j.1463-1326.2001.00126.x

[15] K. Ohno, H. J. Chung, I. Maruyama and T. Tani, "Bofutsushosan, a Traditional Chinese Formulation, Prevents Intimal Thickening and Vascular Smooth Muscle Cell Proliferation Induced by Balloon Endothelial Denudation in Rats,” Biological \& Pharmaceutical Bulletin, Vol. 28, No. 11, 2005, pp. 2162-2165.

http://dx.doi.org/10.1248/bpb.28.2162

[16] M. Kang, J. W. Oh, H. K. Lee, H. S. Chung, S. M. Lee, C. Kim, H. J. Lee, D. W. Yoon, H. Choi, H. Kim, et al., "Anti-Obesity Effect of PM-F2-OB, an Anti-Obesity Herbal Formulation, on Rats Fed a High-Fat Diet,” Biological \& Pharmaceutical Bulletin, Vol. 27, No. 8, 2004, pp. 1251-1256. http://dx.doi.org/10.1248/bpb.27.1251

[17] G. Zheng, M. Jiang, X. He, J. Zhao, H. Guo, G. Chen, Q. Zha and A. Lu, "Discrete Derivative: A Data Slicing Algorithm for Exploration of Sharing Biological Networks between Rheumatoid Arthritis and Coronary Heart Disease,” BioData Mining, Vol. 4, 2011, p. 18. http://dx.doi.org/10.1186/1756-0381-4-18

[18] M. Jiang, C. Zhang, G. Zheng, H. Guo, L. Li, J Yang, C. 
Lu, W. Jia and A. Lu, "Traditional Chinese Medicine Zheng in the Era of Evidence-Based Medicine: A Literature Analysis,” Evidence-Based Complementary and Al- ternative Medicine, Vol. 2012, 2012, Article ID: 409568. 